hep-th/0609148 C Zachos Classical Bound on Quantum Entropy ANL-HEP-PR-06-74

\title{
A CLASSICAL BOUND ON QUANTUM ENTROPY
}

\author{
Cosmas K Zachos \\ \# High Energy Physics Division, Argonne National Laboratory, Argonne, IL 60439-4815, USA \\ zachos@hep.anl.gov
}

\begin{abstract}
A classical upper bound for quantum entropy is identified and illustrated, $0 \leq S_{q} \leq$ $\ln \left(e \sigma^{2} / 2 \hbar\right)$, involving the variance $\sigma^{2}$ in phase space of the classical limit distribution of a given system. A fortiori, this further bounds the corresponding information-theoretical generalizations of the quantum entropy proposed by Rényi.
\end{abstract}

\section{Introduction}

Recurrent problems in four dimensional BPS black holes focus on the entropic behavior of the respective complex structure moduli spaces, and, perhaps independently, on the corresponding holographic entanglement information lost in decoherence, and associated Hawking radiation paradoxes [1]. They all rely on the fundamental and dependable statistical concept of entropy, which accounts collectively for the flow of information in these systems, and for which robust estimates are needed, in lieu of detailed accounts of quantum states. Ideally, such estimates would only require gross geometrical and semiclassical features of the system involved, and ignore quantum mechanical interference subtleties.

Classical continuous distributions have been studied in probability and information theory for quite a long time, and Shannon [2] has derived handy upper bounds for their entropy, and thus crude least information estimates, in the 1940s. Approximate counting of quantum microstates, however, is normally toilsome, and can be approximated heuristically by semiclassical proposals [3], which, ultimately, should devolve to a bona fide classical limit, despite occasional ambiguities and complications along the way [4]. However, a more systematic approach was initiated by Braunss [5], who appreciated the underlying simplicity of phase space in taking a classical limit of intricate quantum systems. He thus tracked the information loss involved in smearing away quantum effects, to argue that the entropy of a quantum system is majorized by that of its classical limit, as $\hbar$ information of the former is forfeited in the latter, an intuitively plausible relation.

The purpose of the present brief remark is to simply combine the two inequalities into a general upper bound of the quantum entropy of a system provided essentially by just the logarithm of the variance in phase space of the classical limit distribution of that system. The resulting inequality, eqn (9) below, is illustrated simply by the elementary physics paradigm of a thermal bath of oscillator 
excitations of one degree of freedom, whose phase-space representation is an obvious maximal entropy Gaussian.

Note that there is no specific assumption of a particular spectral behavior - or even of the existence of a hamiltonian - for the systems covered by the inequality. Extension to arbitrary degrees of freedom and tighter bounds contingent on the circumstances of detailed physical applications are conceptually straightforward, even though specific application to the moduli phase spaces or holographic entanglement of black holes is reserved for a future, less general, report.

In passing, and because it fits naturally with the computational technique involved, the corresponding quantum Rényi entropies [6] are also evaluated explicitly here for the same prototype system, to illustrate the broad fact that these entropies are majorized by the Gibbs-Boltzmann entropy, and thus also by the bound discussed here. Rényi generalized entropies were originally introduced as a measure of complexity in optimal coding theory [6], and have been applied to turbulence, chaos and fractal systems, as well as semi-inclusive multiparticle production [7, 8]; however, apparently, they have not attained significance in black hole physics yet, nor in current noncommutative geometry efforts.

\section{Shannon and Boltzmann-Gibbs entropy in phase space}

For a continuous distribution function $f(x, p)$ in phase space, the classical (Shannon information) entropy is

$$
S_{c l}=-\int d x d p f \ln (f)
$$

For a given distribution function $f(x, p)$, without loss of generality centered at the origin, normalized, $\int d x d p f=1$, and with a given variance, $\sigma^{2}=\left\langle x^{2}+p^{2}\right\rangle=\int d x d p\left(x^{2}+p^{2}\right) f$, it is evident from elementary constrained variation of this $S_{c l}[f]$ w.r.t. $f$, [2] (also see [9]), that it is maximized by the Gaussian, $f_{g}=\exp \left(-\left(x^{2}+p^{2}\right) / \sigma^{2}\right) / \sigma^{2} \pi$, to $S_{c l}=1+\ln \left(\pi \sigma^{2}\right)$.

That is, a Gaussian represents maximal disorder and minimal information - in thermodynamics, least dispersal energy would be available.

Thus, it leads to a standard result in information theory [2], Shannon's inequality,

$$
S_{c l} \leq \ln \left(\pi e \sigma^{2}\right)
$$

which provides an upper bound on the lack of information in such distributions.

Note that, in general, $S_{c l}$ is unbounded above, as it diverges for delocalized distributions, $\sigma \rightarrow \infty$, containing no information. In contrast to the Boltzmann-Gibbs entropy, it is also unbounded below, given ultralocalized peaked distributions $(\sigma \rightarrow 0)$, which reflect complete order and information. 
In quantum mechanics, the sum over all states is given by the standard von Neumann entropy [10] for a density matrix $\rho$,

$$
0 \leq S_{q}=-\operatorname{Tr} \rho \ln \rho=-\langle\ln \rho\rangle
$$

This transcribes in phase space [11, 5] through the Wigner transition map [12] to

$$
0 \leq S_{q}=-\int d x d p f \ln _{\star}(h f)
$$

where the $\star$-product [11]

$$
\star \equiv e^{\frac{i \hbar}{2}\left(\overleftarrow{\partial}_{x} \vec{\partial}_{p}-\overleftarrow{\partial}_{p} \vec{\partial}_{x}\right)}
$$

serves to define $\star$-functions, such as the $\star$-logarithm, above, e.g. through $\star$-power expansions,

$$
\ln _{\star}(h f) \equiv-\sum_{n=1}^{\infty} \frac{(1-h f)_{\star}^{n}}{n}
$$

Braunss [5] has argued that, for $S_{c l}$ defined by $S_{q}+\ln h$ in the limit that the Planck constant $\hbar \rightarrow 0$,

$$
0 \leq S_{q} \leq S_{c l}-\ln h
$$

The logarithmic offset term relying on the Planck constant $h$ accounts for the scale [3] of the phase-space area element $d x d p$ in (4). This scale, $h$, should divide $d x d p$ to yield a dimensionless phase space area element; Correspondingly, it should then multiply $f$, to preserve 'probability', $\int d x d p f=1$, in the Wigner transition map from the density matrix $\rho$ to the Wigner Function $f$. E.g., for a pure state [12],

$$
f(x, p)=\frac{1}{h} \int d y \psi^{*}\left(x-\frac{1}{2} y\right) e^{-i y p / \hbar} \psi\left(x+\frac{1}{2} y\right) .
$$

The classical limit normally entails variations of phase-space variables on scales much larger than $\hbar$. Therefore these variables are normally scaled down to scales matched to such activity. As illustrated explicitly in the next section, comparing quantum and classical entropies relies on the above offset. The upper bound in this Braunss inequality reflects the loss of quantum information involved in the smearing implicit in the classical limit1, effectively regarded as an extreme limit of subadditivity [3].

\footnotetext{
${ }^{1}$ Readers unfamiliar with the classical limit might find loss of the quantum uncertainty of the theory counterintuitive and discordant with the loss of information involved. Actually, the resolution to access the uncertainty is sacrificed in this limit. A standard consequence of the Cauchy-Schwarz inequality for Wigner functions is $|f| \leq 2 / h$, [12], reflecting the uncertainty principle: the impossibility of localizing $f$ in phase space, through a delta function. The best one can do is to take a pillbox cylinder of base $h / 2$ and height $2 / h$, properly normalized to $1=\int d x d p f$. Now, scaling the phase-space variables down and $f$ up (to preserve this normalization - the volume of the pillbox, as in the above discussion of the offset) ultimately collapses the base of the pillbox to a mere point in phase space; and leads to a divergent height for $f$, a delta function, characteristic of a perfectly localized classical particle. However, several different quantum configurations will reduce to this same limit: it is this extra quantum information on $h$-dependent features, e.g. interference, that is obliterated in the limit.
} 
Combined with Shannon's bound, this now amounts to

$$
0 \leq S_{q} \leq \ln \left(\frac{e \sigma^{2}}{2 \hbar}\right)
$$

i.e., the entropy is bounded above by an expression involving the variance of the corresponding classical limit distribution function. It readily generalizes to multidimensional phase space $\left(R^{2 N}\right.$, in which case the logarithm is evidently multiplied by $N$, in evocation of Bekenstein's bound), and contexts where more information (e.g., on asymmetric variances) happens to be available, or refinement desired.

By virtue of (6), the quantum entropy is recognized as an expansion

$$
S_{q}=\sum_{n=1}^{\infty} \frac{\left\langle(1-\rho)^{n}\right\rangle}{n}=\sum_{n=1}^{\infty} \frac{\left\langle(1-h f)_{\star}^{n}\right\rangle}{n} .
$$

The leading term, $n=1, \quad 1-\operatorname{Tr} \rho^{2}=\langle 1-h f\rangle$, is the impurity [11, 10, 12], often referred to as linear entropy. Like the entropy itself, it vanishes for a pure state [11, 10, 12], for which $\rho^{2}=\rho$, or, equivalently, $f \star f=f / h$. Each term in the above expansion then projects out $\rho$, or $\star h f$, respectively: pure states saturate the lower bound on $S_{q}$.

A likewise additive (extensive) generalization of the quantum entropy is the Rényi entropy [6],

$$
R_{\alpha}=\frac{1}{1-\alpha} \ln \left\langle\rho^{\alpha-1}\right\rangle=\frac{1}{1-\alpha} \ln \int \frac{d x d p}{h}(h f)_{\star}^{\alpha},
$$

where the limit $\alpha \rightarrow 1$ yields $R_{1}=S_{q}$, and the above-mentioned impurity is $1-\exp \left(-R_{2}\right)$. For continuous distributions (infinity of components) discussed here, $R_{0}$ is divergent.

For $\alpha \geq 1, R_{\alpha} \geq R_{\alpha+1}$, so $S_{q} \geq R_{\alpha}$, and it is also bounded below by 0 [6], i.e.,

$$
S_{q} \geq R_{\alpha} \geq R_{\alpha+1} \geq 0
$$

so that, a fortiori, the Rényi entropy is also bounded by (9).

\section{Gaussian Illustration}

To illustrate the above inequalities, consider the Gaussian Wigner Function of arbitrary half-variance $E$,

$$
f(x, p, E)=\frac{e^{-\frac{x^{2}+p^{2}}{2 E}}}{2 \pi E}=e^{-\frac{x^{2}+p^{2}}{2 E}-\ln (2 \pi E)} .
$$

This happens to be the phase-space Wigner transform of a Maxwell-Boltzmann thermal distribution for a harmonic oscillator [13], in suitably rescaled units, normalized properly to unity, and with mean energy $E=\left\langle\left(x^{2}+p^{2}\right) / 2\right\rangle$. 
Calculation of the entropy of this distribution, is, of course, a freshman physics problem, but its independent phase-space derivation [14] (also see [15]), is reviewed here, i.e., evaluation of (44) directly.

For $E=\hbar / 2$, the distribution reduces to just $f_{0}$, the Wigner Function for a pure state (the ground state of the harmonic oscillator). Hence [11, 12],

$$
f_{0} \star f_{0}=\frac{f_{0}}{h}
$$

so that $f_{0}$ is $\star$-orthogonal to each of the terms in the sum (6) , and hence $S_{q}=0$, indicating saturation of the maximum possible information content.

For generic width $E$, the Wigner Function $f$ is not that of a pure state, but it still happens to always amount to a $\star$-exponential [16] $\quad\left(e_{\star}^{a} \equiv 1+a+a \star a / 2 !+a \star a \star a / 3 !+\ldots\right)$ as well,

$$
h f=e^{-\frac{x^{2}+p^{2}}{2 E}+\ln (\hbar / E)}=e_{\star}^{-\frac{\beta}{2 \hbar}\left(x^{2}+p^{2}\right)+\ln \left(\frac{\hbar}{E} \cosh (\beta / \hbar)\right)},
$$

where an "inverse temperature" variable $\beta(E, \hbar)$ is useful to define,

$$
\tanh (\beta / 2) \equiv \frac{\hbar}{2 E} \leq 1 \quad \Longrightarrow \quad \beta=\ln \frac{E+\hbar / 2}{E-\hbar / 2}
$$

(Thus the above pure state $f_{0}$ corresponds to zero temperature, $\beta=\infty$.)

Since $\star$-functions, by virtue of their $\star$-expansions, obey the same functional relations as their non$\star$ analogs, inverting the $\star$-exponential through the $\star$-logarithm and integrating (44) yields directly the standard thermal physics result,

$$
S_{q}(E, \hbar)=\frac{E}{\hbar} \ln \left(\frac{2 E+\hbar}{2 E-\hbar}\right)+\frac{1}{2} \ln \left(\left(\frac{E}{\hbar}\right)^{2}-\frac{1}{4}\right)=\frac{\beta}{2} \operatorname{coth}(\beta / 2)-\ln (2 \sinh (\beta / 2)) .
$$

Indeed, this can be seen to be a monotonically nondecreasing function of $E$, attaining the lower bound 0 for the pure state $E \rightarrow \hbar / 2(\beta \rightarrow \infty$, zero temperature).

The classical limit, $\hbar \rightarrow 0(\beta \rightarrow 0$, infinite temperature) thus follows,

$$
S_{q} \rightarrow 1+\ln (E / \hbar)=\ln (\pi e 2 E)-\ln h=S_{c l}(E)-\ln h,
$$

and is explicitly seen to bound the expression (17) for all $E$, saturating it for large $E>>\hbar$, in accordance with Braunss' bound. That is, the upper bound (9) is saturated for Gaussian quantum Wigner functions with $\sigma^{2}>>\hbar$.

Note the region $E<\hbar / 2$, corresponding to ultralocalized spikes excluded by the uncertainty principle, was not allowed by the above derivation method, since, in this region, no $\star$-Gaussian can be found to represent the Gaussian. (It would amount to complex $\beta$ and $S_{q}$, linked to thermal expectations of the oscillator parity operator.)

NB. An alternate heuristic proposal of ref [3] for the classical limit of the entropy effectively starts from the Husimi phase-space representation [12]; it first effectively drops all $\star_{H} \mathrm{~S}$ in (4) and easily 
evaluates (11) instead (which is well-defined because $f_{H} \geq 0$ automatically), before completing the transition to the classical limit $\hbar \rightarrow 0$. It also, ultimately, yields the same answer (18), since the Husimi representation of the Gaussian Wigner Function (13),

$$
f_{H} \equiv \int d x^{\prime} d p^{\prime} \frac{e^{-\left(\left(x^{\prime}-x\right)^{2}+\left(p^{\prime}-p\right)^{2}\right) / \hbar}}{\pi \hbar} f\left(x^{\prime}, p^{\prime}\right)=\frac{e^{-\frac{x^{2}+p^{2}}{2 E+\hbar}}}{\pi(2 E+\hbar)}
$$

is also a Gaussian. Utilized to evaluate (1), it yields $\ln (\pi e(2 E+\hbar))$, which has the more direct expression $S_{c l}$ of (18) as its classical limit. (For the ground state, $E=\hbar / 2$, which is a coherent state, this semiclassical entropy reduces to a characteristic minimal value, $1+\ln h$.)

By virtue of (15), *-powers of the Gaussian are also straightforward to take, and thus the Rényi entropies can be readily computed:

$$
R_{\alpha}=\frac{1}{1-\alpha} \ln \left(\frac{(2 \sinh (\beta / 2))^{\alpha}}{2 \sinh (\alpha \beta / 2))}\right)=\frac{1}{\alpha-1} \ln \left(\left(\frac{E}{\hbar}+\frac{1}{2}\right)^{\alpha}-\left(\frac{E}{\hbar}-\frac{1}{2}\right)^{\alpha}\right) .
$$

Note $\alpha \rightarrow 1$ checks with the above (17), $R_{1} \rightarrow S_{q}$. Also, in the pure state limit, $E=\hbar / 2$, it is evident that $R_{\alpha}=0$ checks for all $\alpha \geq 1$. (For $\alpha>1$ and the small disallowed values $E<\hbar / 2$, $R_{\alpha}<0$.)

$R_{\alpha}$ is also a nondecreasing function of $E$; and, in comportance with (12), a nonincreasing function of $\alpha$. Up to an additive, $\alpha$-dependent constant, the classical limit is identical to that for the entropy itself,

$$
R_{\alpha} \rightarrow \frac{\ln \alpha}{\alpha-1}+\ln (E / \hbar)
$$

in agreement with the classical result of [8]. It may well be that, as in the contexts touched upon in the introduction, specific $\alpha$ s may well provide more detailed or practical measures of complexity in Hawking radiation with sparse information available.

If a specific quantum Hamiltonian were actually available for the system in question (a rare occurrence), then the classical limit of the entropy of the system would be straightforward - and thus the inequality discussed here would not be that powerful, since the classical entropy itself would be at hand, in general lower than the Shannon bound.

For such a simple system, the upper-bounding classical entropy would result out of the phasespace partition function specified by the corresponding classical hamiltonian (the Weyl symbol of the quantum hamiltonian). This is easily illustrated explicitly by hamiltonians which are positive $N$-th powers of the oscillator hamiltonian, so that, simply,

$$
f_{c l} \propto \exp \left(-\left(\left(x^{2}+p^{2}\right) / 2 E\right)^{N}\right)
$$

The bounding classical entropy then reduces by standard thermodynamic evaluation to be just (1),

$$
S_{c l}=\frac{1}{N}+\ln \left(2 \pi E \Gamma\left(1+\frac{1}{N}\right)\right)
$$


lower than the cooresponding Shannon bound,

$$
1+\ln \left(\pi E \frac{\Gamma(1+2 / N)}{\Gamma(1+1 / N)}\right) .
$$

Note Added A referee has identified a technical gap in Braunss' formal proof of his inequality in [5], which is, nevertheless, assumed here.

This work was supported by the US Department of Energy, Division of High Energy Physics, Contract DE-AC02-06CH1135\%; and the Collaborative Project GEP1-3327-TB-03 of the US Civilian Research and Development Foundation. Helpful discussions with A Polychronakos, T Curtright, and $G$ Jorjadze are acknowledged.

\section{References}

[1] H Ooguri, A Strominger, and C Vafa, Phys Rev D70 (2004) 106007, [hep-th/0405146];

G L Cardoso, D Lüst, and J Perz, JHEP 0605 (2006) 028, [hep-th/0603211];

V Balasubramanian, J de Boer, V Jejjala, and J Simon, JHEP 0512 (2005) 006, hep-th/0508023

A Strominger and D Thompson, Phys Rev D70 (2004) 044007, [hep-th/0303067];

T Hirata and T Takayanagi, JHEP 0702 (2007) 042, hep-th/0608213];

C Gomez and S Montanez, JHEP 0612 (2006) 069, hep-th/0608162

[2] R B Ash, Information Theory (Dover, NY, 1965);

C Shannon, Bell Syst Tech J 27 (1948/9) 379-423; 623-656

[3] A Wehrl, Rev Mod Phys 50 (1978) 221-260; Rep Math Phys 16 (1979) 353-358

[4] T Takabayasi, Prog Theo Phy 11 (1954) 341-373;

G P Beretta, J Math Phys 25 (1984) 1507-1510;

G Manfredi and M Feix, Phys Rev E62 (2000) 4665-4674

[5] G Braunss, J Math Phys 35 (1994) 2045-2056

[6] A Rényi, Probability Theory (North Holland, Amsterdam, 1970), pp 574,579,580;

J Włodarz, Int J Theor Phys 42 (2003) 1075-1084

[7] P Jizba and T Arimitsu, Phys Rev E69 (2004) 026128;

A Bialas, W Czyz, and K Zalewski, Phys Rev C73 (2006) 034912

[8] I Varga and J Pipek, Phys Rev E68 (2003) 026202

[9] A K Rajagopal, Phys Rev A27 (1983) 558-561

[10] U Fano, Rev Mod Phys 29 (1957) 74-93

[11] H J Groenewold, Physica 12 (1946) 405-460; and Phys Repts 11C (1974) 327-354 
[12] C Zachos, D Fairlie, and T Curtright, Quantum Mechanics in Phase Space: an Overview with Selected Papers (World Scientific Publishers, Singapore, 2005)

[13] M Bartlett and J Moyal, Proc Camb Phil Soc 45 (1949) 545-553

[14] Lipo Wang, J Math Phys 27 (1986) 483-487

[15] G S Agarwal, Phys Rev A3 (1971) 828-831

[16] K Imre, K Ozizmir, M Rosenbaum, and P Zweifel, J Math Phys 8 (1967) 1097-1108 